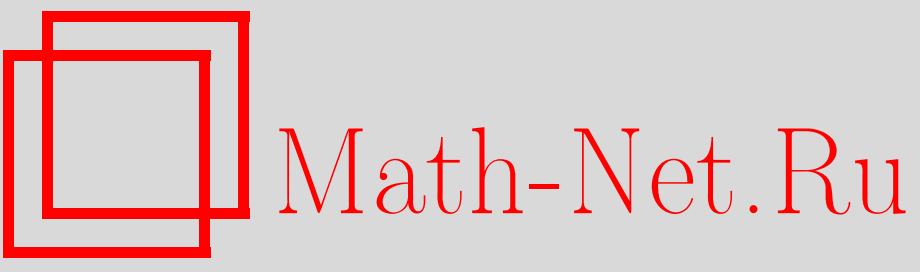

А. М. Райгородский, О размерности в проблеме Борсука, УМH, 1997, том 52, выпуск 6, 181-182

DOI: https://doi.org/10.4213/rm912

Использование Общероссийского математического портала Math-Net.Ru подразумевает, что вы прочитали и согласны с пользовательским соглашением

http://www.mathnet.ru/rus/agreement

Параметры загрузки:

IP : 54.224 .187 .69

26 апреля 2023 г., 12:56:19 


\title{
О РАЗМЕРНОСТИ В ПРОБЛЕМЕ БОРСУКА
}

\author{
А. М. РАЙГОРОДСКИЙ
}

1. Введение. В [1] Борсук высказал гипотезу о том, что всякое ограниченное множество в $\mathbb{R}^{d}$ может быть разбито на $d+1$ часть меньшего диаметра. Определим величину $f(d)$ как минимальное число такое, что всякое ограниченное множество в $\mathbb{R}^{d}$ может быть разбито на $f(d)$ частей меньшего диаметра. Утверждение гипотезы Борсука было доказано в размерностях 2 и 3 , а также в произвольной размерности для множеств с гладкой границей. В работе [2] с помощью результатов работы [3] была получена оценка $f(d) \geqslant(1.2)^{d^{1 / 2}}$, которая позволила построить контрпример к гипотезе Борсука в размерности 1325. В [4] контрпример был построен в размерности $d=946$. В настоящей работе за счет уточнения техники, предложенной в работе [4], доказьвается следующее

УтВеРЖДЕнИЕ. При $d=561$ существует ограниченное множество в $\mathbb{R}^{d}$, которое не может быть разбито на $d+1$ часть меньшего диаметра.

2. Доказательство утверждения. Положим $n=36$. Определим семейство векторов $\Sigma \subset \mathbb{R}^{n}$ как множество всевозможных вершин куба $[-1,1]^{n}$, у которого первые три координаты положительны, а число отрицательных координат четно. Ясно, что $\operatorname{card} \Sigma=2^{n-4}$. Кроме того, легко видеть, что выполнены следующие 3 простые утверждения:

(i) для любых двух векторов $\mathbf{a}, \mathbf{b} \in \Sigma(\mathbf{a}, \mathbf{b}) \equiv 0(\bmod 4)$;

(ii) для любых двух различных векторов $\mathbf{a}, \mathbf{b} \in \Sigma(\mathbf{a}, \mathbf{b}) \equiv 0(\bmod 9)$ тогда и только тогда, когда $(\mathbf{a}, \mathbf{b})=0$;

(iii) для любых двух векторов $\mathbf{a}, \mathbf{b} \in \Sigma(\mathbf{a}, \mathbf{b}) \equiv 4(\bmod 9)$ тогда и только тогда, когда $(\mathbf{a}, \mathbf{b})=4$.

Каждому вектору $\mathbf{a} \in \Sigma$ поставим в соответствие многочлен $F_{\mathbf{a}} \in \mathbf{Q}\left[x_{4}, \ldots, x_{n}\right]$, определяемьй соотношением $F_{\mathbf{a}}(x)=\frac{1}{9} \prod_{i=1}^{3}(i-(\mathbf{a}, \mathbf{x})) \prod_{i=5}^{8}(i-(\mathbf{a}, \mathbf{x}))$. (Здесь предполагается, что $\mathbf{x}=$ $\left(x_{1}, \ldots, x_{n}\right) \in \mathbb{R}^{n}$ и $x_{1}=x_{2}=x_{3}=1$.) Из определения многочлена немедленно следует, что для любых двух векторов $\mathbf{a}, \mathbf{x} \in \Sigma$ выполнено:

(1) $F_{\mathbf{a}}(\mathbf{x}) \in \mathbb{Z}$

(2) если $(\mathbf{a}, \mathbf{x}) \equiv 0(\bmod 9)$, то $F_{\mathbf{a}}(\mathbf{x}) \not \equiv 0(\bmod 3)$;

(3) если $(\mathbf{a}, \mathbf{x}) \not \equiv 0(\bmod 9)$ и $(\mathbf{a}, \mathbf{x}) \not \equiv 4(\bmod 9)$, то $F_{\mathbf{a}}(\mathbf{x}) \equiv 0(\bmod 3)$.

Используя стандартное представление многочлена $F_{\mathbf{a}}$ в виде линейной комбинации мономов и последовательно применяя соотношение $x_{i}^{2}=1$, для каждого вектора $\mathbf{a} \in \Sigma$ получаем новьй многочлен $\widetilde{F}_{\mathbf{a}} \in \mathbf{Q}\left[x_{4}, \ldots, x_{n}\right]$. Понятно, что и для любого такого многочлена выполняются условия (1)-(3).

Рассмотрим произвольне семейство векторов $Q \subset \Sigma$ такое, что скалярное произведение любых двух его различных векторов не ноль и не четыре по модулю 9.

Лемма. Каково бы ни было рассмотренное выше семейство векторов $Q \subset \Sigma$, для величины $s=\operatorname{card} Q$ выполнена оченка:

$$
s \leqslant \sum_{k=0}^{7} C_{33}^{k} .
$$

Настоящая работа выполнена при финансовой поддержке Российского фонда фундаментальных исследований (грант № 96-01-00378). 
ДокАЗАТЕЛЬСтво. Положим $Q=\left\{\mathbf{a}_{1}, \ldots, \mathbf{a}_{s}\right\}$. Каждому вектору $\mathbf{a}_{i} \in Q$ сопоставим многочлен $\widetilde{F}_{\mathbf{a}_{1}}$. Используя свойства $(1),(2),(3)$ многочленов $\widetilde{F}_{\mathbf{a}_{1}}, \ldots, \widetilde{F}_{\mathbf{a}_{s}}$, нетрудно показать аналогично тому, как это было сделано в работе [4], что многочлены $\widetilde{F}_{\mathbf{a}_{1}}, \ldots, \widetilde{F}_{\mathbf{a}_{s}}$ линейно независимы над $\mathbf{Q}$. Вспоминая определение многочленов $\widetilde{F}_{\mathbf{a}_{1}}, \ldots, \widetilde{F}_{\mathbf{a}_{s}}$, получаем, что $s \leqslant \sum_{k=0}^{7} C_{33}^{k}$. Лемма доказана.

Семейству векторов $\Sigma$ сопоставим семейство $(n-1)(n-3)$-мерных векторов $\Sigma^{*}$ следующим образом. Каждому вектору $\mathbf{x} \in \Sigma$ поставим в соответствие вектор $\mathbf{x} * \mathbf{x}=\left(x_{i} \times x_{j}\right), i=2, \ldots, n$, $j=4, \ldots, n$. Легко убедиться в том, что для любых двух векторов $\mathbf{x} * \mathbf{x}, \mathbf{z} * \mathbf{z} \in \Sigma^{*}$ вьполнено равенство $(\mathbf{x} * \mathbf{x}, \mathbf{z} * \mathbf{z})=((\mathbf{x}, \mathbf{z})-1)((\mathbf{x}, \mathbf{z})-3)$. Отсюда и из свойства (i) следует, что максимальное расстояние между векторами из $\Sigma^{*}$ достигается в том и тольо том случае, когда скалярное произведение их прообразов равно нулю или четырем. Воспользовавшись последним рассуждением, утверждениями (ii) и (iii) и неравенством $(*)$, получаем, что множество $\Sigma^{*}$ невозможно разбить на $f<2^{32} /\left(\sum_{k=0}^{7} C_{33}^{k}\right)$ частей так, чтобы диаметр каждой части был меньше диаметра всего множества. Кроме того, понятно, что размерность семейства векторов $\Sigma^{*}$ равна $C_{33}^{2}+33=561$. Положим $d=561$. Из всего сказанного следует, что $f(d) \geqslant 2^{32} /\left(\sum_{k=0}^{7} C_{33}^{k}\right) \geqslant 758>562$. Этим неравенством построение контрпримера к гипотезе Борсука в размерности $d=561$ завершено.

Автор благодарит А. С. Тарасова за указание неточностей в первоначальном варианте работы.

\section{СПИСОК ЛИТЕРАТУРЫ}

[1] Borsuk K. // Fund. Math. 1933. V. 20. P. 177-190. [2] Kahn J., Kalai G. // Bull. AMS (N.S.). 1993. V. 29. № 1. P. 60-62. [3] Frankl P., Wilson R. // Combinatorica. 1981. № 1. P. 259-286. [4] Nilli A. // Contemp. Math. 1994. V. 178. P. 209-210.

Московский государственньй

Принято редколлегией университет им. М. В. Ломоносова 03.09.1997 